\title{
Identification of Preclinical Markers Related to Hereditary Diseases: Expanding the Horizons of the Study of Cardiac Autonomic Modulation
}

\author{
Nágela Nunes ${ }^{1,2}$ and Paulo R. Benchimol-Barbosa ${ }^{1,3}$ \\ Complexo Hospitalar de Niterói, ${ }^{1}$ Niterói, RJ - Brazil. \\ Hospital Universitário Antônio Pedro, ${ }^{2}$ Niterói, $R J-$ Brazil. \\ Hospital Universitário Pedro Ernesto, ${ }^{3}$ Rio de Janeiro, RJ - Brazil. \\ Editorial related to the article: Influence of Family History of Diabetes on Cardiac Autonomic Dysfunction of Adolescents
}

The Autonomic Nervous System (ANS) consists of two opposite pathways (sympathetic and parasympathetic) that have action both on the atrial and ventricular myocardium, as well as on atrioventricular and sinus nodes, thus exerting influence on the variation of heart rate (HR). The increase in HR is a consequence of the greater action of the sympathetic pathway and the lower parasympathetic activity (vagal inhibition), while its reduction basically depends on predominance of vagal activity. The influence of ANS on the heart is dependent on information that departs, among others, from baroceptors, chemoreceptors, changes in the respiratory system, vasomotor system, renin-angiotensinaldosterone system and thermoregulatory system.

The discovery of the relationship between ANS and mortality from cardiovascular diseases has led to several studies, which have proven the existence of reduced parasympathetic and increased sympathetic activity in several pathologies of the cardiovascular system. These findings made it necessary to develop quantitative markers of cardiac autonomic activity, and heart rate variability (HRV) is the most promising autonomic marker ${ }^{1}$. Historically, their clinical interest arose in 1965, when Hon and Lee demonstrated a well-defined clinical application of HRV in the area of fetal distress monitoring. In 1977, Wolf et al., ${ }^{2}$ showed an association between low HRV and higher risk of mortality after acute myocardial infarction and Kleiger et al., ${ }^{3}$ in 1987,

\section{Keywords}

Autonomic Nervous System/complications; Diabetes Mellitus/complications; Autonomic Denervation; Genetic Diseases, Inborn; Adolescent. confirmed that HRV was a potent and independent predictor of mortality after acute myocardial infarction. In 1996, in an age-stratified cross-sectional study, Barbosa et al., ${ }^{4}$ reported $>18 \%$ per decade reduction in HRV in healthy adults and $>25 \%$ per decade reduction in subjects two years post-myocardial infarction with persevered left ventricular systolic function. ${ }^{5}$ Additionally, the authors observed that aging related HRV decrease was mainly related to reduction in spontaneous vagal activity. In 2002, Nunes studied HRV variability in preschool healthy children, defining normality ranges for HRV variables in this population. ${ }^{6}$

HRV represents the spontaneous and continuous variation of cardiac interbeat interval. Therefore, HRV comprises the oscillations between the RR intervals of heartbeat, which reflect the changes resulting from the action of the ANS on the behavior of HR. HRV's analyses, from linear methods, can be performed as a function of two parameters: time domain (analysis of records deriving from times greater than 10 minutes, expressed in milliseconds, through variation of the duration of intervals between normal QRS complexes resulting from sinus depolarization and its mathematical indices) and frequency domain (records of wave intensity verified in time intervals of up to 4 minutes and it's unit is determined by Hertz).

In the time domain, SDNN and SDNNi are indexes taken through individual RR intervals and represent sympathetic and parasympathetic activities, while rMSSD and pNN50 are obtained through adjacent RR intervals and reflect parasympathetic activity. The parameters obtained by the frequency domain are: HF (High Frequency): values between 0,15 a $0,4 \mathrm{~Hz}$, related to respiratory activity and it is an indication 
of vagus influence on the heart; LF (Low Frequency): associated with baroreceptor reflex with values between 0.04 and $0.15 \mathrm{~Hz}$, resulting from the joint action of the parasympathetic and sympathetic components on the heart, with predominance of sympathetic; LF/HF ratio is calculated and provide sympathetic / parasympathetic balance. The limitation of implementation this technique includes the presence of all no sinus rhythm, heart transplant, pacemaker activity and the presence of atrioventricular block.

The measurement of HRV is extremely important for clinical understanding of physiological variables, since the increase in HRV indicates good physiological adaptation, while the reduction has been pointed out as a predictor of diseases or the occurrence of adverse events in patients with previous diseases. Numerous conditions have been described as responsible for measurable autonomic changes through HRV, such as: several heart diseases, nervous anorexia, epilepsy, asthma, anxiety disorders, obesity, hypertension and diabetes mellitus.

Diabetes mellitus (DM) is a global health epidemic thought to be affecting 415 million people worldwide, with a further 318 million suffering with glucose intolerance and at increased risk of developing the disease. Type II DM is a multifactorial polygenic inheritance form of diabetes, comprising about $90 \%$ all DM cases. About $75 \%$ may manifest sustained elevated blood glucose levels as early as in the fifth decade of life. Cardiac autonomic neuropathy (CAN) is a common underdiagnosed complication of DM. ${ }^{7}$ Interestingly, subtle autonomic neurologic deficits have also been documented earlier in the course of diabetes and during the prediabetic period in subjects with impaired glucose tolerance in comparison with healthy individuals. The impact of CAN on patients with DM can be devastating, and it has been shown that impaired autonomic system can be associated with increased mortality, cardiovascular disease (CVD), chronic kidney disease (CKD), and morbidity in DM. CAN has several risk factors that are common to other diabetes-related vascular complications, such as: glycemic control, diabetes duration and CVD risk factors. Besides that, several genes have been linked to the development and progression of diabetic polyneuropathy and CAN, among which are TCF9L2, APOE, and ACE. ${ }^{8}$

CAN, usually, only becomes symptomatic in the later stages of the disease and the vagus nerve is usually the first nerve to be affected, resulting in symptoms of sympathetic predominance. Subclinical CAN is initially limited to baroreceptor abnormalities and reduction in HRV, but as the disease progresses, cardiac involvement becomes more evident and symptomatic with resting tachycardia, reduced exercise tolerance, orthostatic hypotension, QT prolongation, silent ischemia, cardiomyopathy and CKD. ${ }^{6}$

Early determination of CAN is vital to the success of therapeutic input, as it has been suggested that cardiovascular denervation may be reversible if diagnosed soon after onset, through a combination of nonpharmacological and pharmacological approaches, including lifestyle modification, intensive glycemic control, and treating underlying risk factors, such as hyperlipidemia and hypertension. ${ }^{9}$

Dias-Filho et al. ${ }^{10}$, conducted a Brazilian multicenter study to evaluate cardiac autonomic modulation of adolescents with a family history of diabetic parents. They selected 69 teenagers from state public schools, aged 11 to 18 years, 23 with family history of diabetes. The authors managed to investigate nonmetabolic traits of early putative phenotypic expression of diabetes, specifically on the autonomic modulation of the heart. They have found that teenagers with parental history of diabetes, as compared to those with no such familial history, showed slight but significant overall reduction in heart rate variability, with a particular effect on the vagal limb of heart rate modulation.

The study protocol consisted of the analysis of heart rate variability, blood pressure, anthropometric measurements, and body composition. They also used questionnaires to evaluate level of physical activity, sexual maturation, and sleep quality and all variables were paired with no significant difference between the 2 groups. When the group of individuals with a family history of diabetes was compared with the control group, statistically significant differences were observed in SDNN ( $43.9 \pm 2.2$ vs. $53.5 \pm 2.6 \mathrm{~ms})$, RMSSD $(41.9 \pm 3.3$ vs. $52.4 \pm 3.2 \mathrm{~ms})$, SD1 $(29.7 \pm 2.3$ vs. $37.1 \pm 2.3 \mathrm{~ms})$, SD2 $(54.1 \pm 2.6$ vs. $66.66 \pm 3.5 \mathrm{~ms})$, and LF $(496.0 \pm 49.5$ vs. 728 $\left.\pm 71.6 \mathrm{~ms}^{2}\right)$ and HF $\left(1050.0 \pm 120.4\right.$ vs. $\left.737.4 \pm 98.5 \mathrm{~ms}^{2}\right)$. They concluded that global autonomic modulation is decreased in adolescents with a family history of diabetes.

These important findings suggest an early decrease in vagal tone in adolescents with diabetic parents, suggesting that the sympathetic / vagal imbalance in this population could be an earlier mark of disease and might be considered to optimize non-pharmacological measures that would prevent the development of diabetes in the future. 
Some considerations, however, deserve to be highlighted. It is important to stress that present findings do not allow one to infer the presence of disease or dysfunction in healthy teenager population. All variables showed results within expected normal limits, although significant differences were found between groups. The paired data table of adolescent populations in the study does not inform whether there was also a pairing regarding gender, since it is known that sex can influence HRV. In 2006, Rajendra Acharya et al., ${ }^{11}$ observed that

\section{References}

1. Lopes P, Oliveira M, André S, Nascimento DLA, Silva SSS, Rebouças GM, et al.et al. Aplicabilidade Clínica da Variabilidade da Frequência Cardíaca. Rev Neurociênc. 2014;21(4):600-3.

2. Wolf MM, Varigos G A, Hunt D SJG. Sinus Arrhythmia in Acute Myocardial Infarction .Med J Aust. 1978;52-3.

3. Kleiger RE, Miller JP, Bigger JT, Moss AJ. Decreased heart rate variability and its association with increased mortality after acute myocardial infarction. Am J Cardiol 1987;59(4):256-62.

4. Vanderlei LCM, Pastre CM, Hoshi RA, de Carvalho TD, de Godoy MF. Noções básicas de variabilidade da frequência cardíaca e sua aplicabilidade clínica. Brazilian J Cardiovasc Surg. 2009;24(2):205-17.

5. Barbosa PR, Barbosa Filho J, Sá CAM. Influência da Idade, Sexo e Doença Coronária sobre a Modulação Autonômica do Coração. Arq Bras Cardiol. 1996; 67(5): 325-9

6. Nunes NSV. [Contribution to the study of heart rate variability in children without evidences of structural heart disease]. Tese. Rio de Janeiro:Universidade Federal Fluminense, (Cardiologia Clínica);2002.
HRV is lower with age and that variation is greater in women, particularly during and preceding the menstrual period. It is also necessary when analyzing HRV in a population, that all variables that might affect it in some way should be controlled. In addition to gender, variables such as major or minor component of REM sleep, presence or absence of sleep apnea, would be more useful than just the sleep quality questionnaire, which, in addition to being flawed, does not report relevant sleep data that can influence HRV. Finally, due to the small sample size, these findings must be confirmed later.

7. Karayannis G, Giamouzis G, Cokkinos D V., Skoularigis J, Triposkiadis F. Diabetic cardiovascular autonomic neuropathy: Clinical implications. Expert Rev Cardiovasc Ther. 2012;10(6):747-65.

8. Fisher VL, Tahrani AA. Cardiac autonomic neuropathy in patients with diabetes mellitus: Current perspectives. Diabetes, Metab Syndr Obes Targets Ther. 2017;10:419-34.

9. Balcıoğlu AS. Diabetes and cardiac autonomic neuropathy: Clinical manifestations, cardiovascular consequences, diagnosis and treatment. World J Diabetes. 2015;6(1):80.

10. Dias-Filho CA, Soares Jr NJ, Dias CJ, et. al. Influence of Family History of Diabetes on Cardiac Autonomic Dysfunction of Adolescents. Int J Cardiovasc Sci. 2020; 33(4):360-367. DOI: 10.36660/ijcs.20180064

11. Acharya UR, Joseph KP, Kannathal N, Lim CM, Suri JS. Heart rate variability: A review. Med Biol Eng Comput 2006;44(12):1031-51. 